\title{
UCLA Neptune Facility for Advanced Accelerator Studies
}

\author{
Sergei Ya. Tochitsky, ${ }^{1}$ Christopher E. Clayton, ${ }^{1}$ Kenneth A. Marsh, ${ }^{1}$ \\ James B. Rosenzweig, ${ }^{2}$ Claudio Pellegrini ${ }^{2}$ and Chandrashekhar Joshi ${ }^{1}$ \\ Neptune Laboratory, ${ }^{1}$ Department of Electrical Engineering, ${ }^{2}$ Department of Physics, University of \\ California at Los Angeles, 405 Hilgard avenue, Los Angeles, CA 90095
}

\begin{abstract}
The Neptune Laboratory at UCLA is being used for exploring concepts useful for advanced accelerators. This facility hosts a TW-class $\mathrm{CO}_{2}$ laser system and a high-brightness photoinjector producing a $14 \mathrm{MeV}$ electron beam. The goal for the laboratory is to carry out experiments on high-gradient acceleration of externally injected electrons in both laser-driven relativistic plasma waves and EM laser field in vacuum. Experiments on plasma beat-wave acceleration using a prebunched electron beam, a high-energy gain 10- $\mu \mathrm{m}$ inverse free electron laser accelerator, longitudinal electron beam shaping and laser based light-sources are planned.
\end{abstract}

\section{INTRODUCTION}

The future of high-energy physics will depend on the development of accelerator technology, which can provide acceleration gradients well in excess of $100 \mathrm{MeV} / \mathrm{m}$ (the field emission limit for a copper RF structure). The electric field of a laser at its focus can exceed this value by many orders of magnitude. As a result, many schemes for coupling the energy of a transverse electromagnetic wave of the laser to charged particles were proposed and high-gradient laser-driven particle accelerators were successfully demonstrated both in plasmas [1] and in vacuum [2]. In spite of the demonstrated high gradients, the potential of laser-driven particle accelerators for high-energy physics applications cannot be ascertained without solving the issue of matching of a particle beam into the laser-driven accelerating structure and efficient extraction of this beam from such a structure. This is important for optimizing the energy extraction efficiency as well as for staging these laser driven structures.

The Neptune Laboratory is constructed at UCLA to address the issues of beam loading by injecting a well-characterized electron beam into a preformed plasma accelerating structure or a magnetic undulator and explore methods for extracting a high-quality beam from such a structure. This facility hosts a TW-class $\mathrm{CO}_{2}$ laser system and a high-brightness photoinjector producing a $14 \mathrm{MeV}$ electron beam. As of now the electrons were injected in a plasma beat wave accelerator (PBWA) with a relativistic plasma wave excited over several centimeters [3]. Also the availability of this high-power $10-\mu \mathrm{m}$ beam has recently made possible inverse free electron laser (IFEL) acceleration in vacuum [4]. In this paper we first present a description of the Neptune facility and then review main experimental results and plans for future work. 


\section{NEPTUNE FACILITY}

The Neptune Laboratory comprises two main components, the TW-class $\mathrm{CO}_{2}$ laser system and the RF photoinjector as well as various diagnostic tools for the laser and the electron beams. At present the state-of-the-art two-wavelength, $\mathrm{CO}_{2}$ laser system, based on multistage amplification of a 100 ps pulse, is built and fully characterized. The system is capable of producing $100-400$ ps pulses with energy $100-$ $200 \mathrm{~J}$ [5]. The $\mathrm{CO}_{2}$ laser chain includes a two-wavelength hybrid TEA master oscillator, an UV-preionized, 8-atm regenerative amplifier, and a 2.5-atm electronbeam-controlled large-aperture amplifier. The laser beam can be focused down to a spot size of $35 \mu \mathrm{m}$ reaching an intensity of $\sim 10^{16} \mathrm{~W} / \mathrm{cm}^{2}$. The Neptune RF gun and LINAC produce $14 \mathrm{MeV}, 10 \mathrm{ps}$ (FWHM) electron bunches with an emittance of 6 $\pi \mathrm{mm}$ mrad [6] with a typical charge of around $200 \mathrm{pC}$.

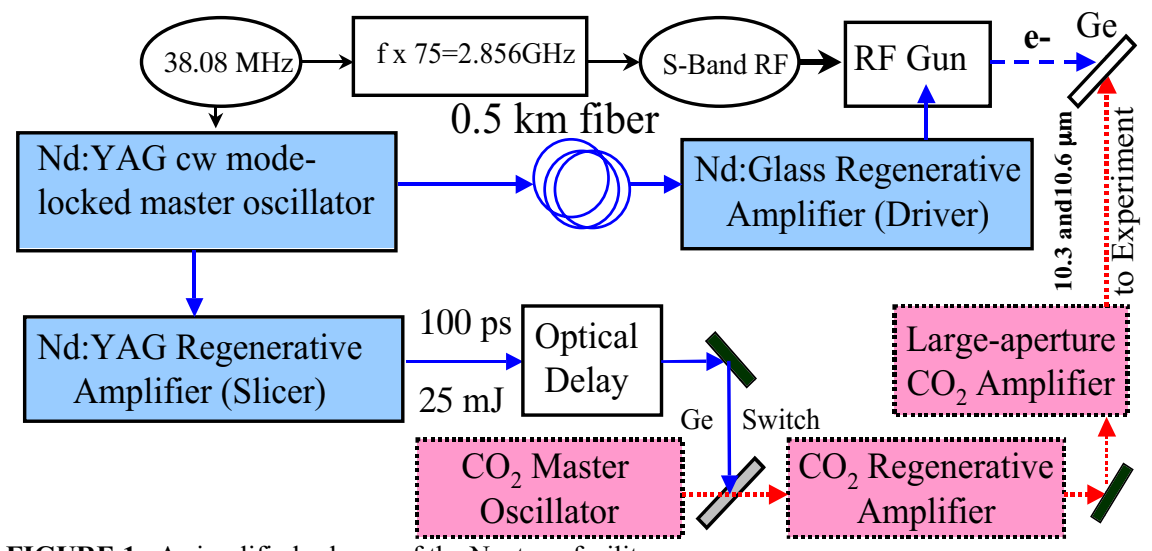

FIGURE 1. A simplified scheme of the Neptune facility.

As shown in Fig.1 a low level RF starts with the $38.08 \mathrm{MHz}$ output signal of a local crystal oscillator controlling an actively mode-locked Nd:YAG master oscillator. This signal is frequency multiplied by 75 to generate S-Band RF. A $100 \mathrm{ps}$ seed pulse produced by the Nd:YAG oscillator is chirped and stretched in a $0.5 \mathrm{~km}$ long fiber, amplified in a Nd:Glass regenerative amplifier (Driver) and then compressed down to 3-10 ps (FWHM) by a grating pair. The fourth harmonic of the $1-\mu \mathrm{m}$ pulse is sent to the photocathode of the RF gun and produces a short, 10 ps electron bunch. The same $\mathrm{Nd}$ :YAG oscillator pulse is used, after amplification in a Nd:YAG regenerative amplifier (Slicer), for slicing a short seed pulse for $10 \mu \mathrm{m} \mathrm{CO}$ amplifiers. To achieve this, the nanosecond $\mathrm{CO}_{2}$ master-oscillator output is combined with the $100 \mathrm{ps}, 1 \mu \mathrm{m}$ pulse on the surface of a semiconductor material [5]. This semiconductor switching technique also provides the ability to synchronize the short $10-\mu \mathrm{m}$ pulse and the electron bunch, since the same Nd:YAG oscillator pulse is used for their production. As a result of such optical locking nanosecond synchronization is achieved electronically by controlling the number of round trip passes in the Slicer laser while an optical delay line provides picosecond synchronization. The latter requires timeresolved measurements with $\sim 10 \mathrm{ps}$ resolution at the point where experiments take 
place. This is realized by the cross-correlation between the photons and electrons using an electron-beam-controlled transmission of the unamplified $10 \mu \mathrm{m}$ pulse in Ge [3]. A total uncertainty of $20 \mathrm{ps}$ is achieved through this synchronization. The residual time jitter is mainly related to fluctuations in the group velocity of the laser pulse in the final $\mathrm{CO}_{2}$ amplifier. Recently we further advanced the synchronization technique at the Neptune Laboratory by setting up an in situ measurement of the relative photoelectron timing for each data shot using a dedicated streak camera [4].

\section{Present Parameters and Proposed Upgrade}

The parameters of the Neptune $\mathrm{CO}_{2}$ laser system and the photoinjector are summarized in Tables 1 and 2. Three main objectives stand behind the proposed upgrade of the main components of the Neptune facility (see Tables 1 and 2):

1) An upgrade of key parameters of both the $\mathrm{CO}_{2}$ laser system and the photoinjector will allow several new experiments (discussed in the next section) to be carried out.

2) A significant improvement in stability and reproducibility of both the laser pulse and the electron beam will increase data acquisition efficiency critical for single-shot measurements and therefore will enable some experiments.

3) By eliminating several outdated laser subsystems, we minimize the very real risk of major down-time that may jeopardize the planned experimental program.

TABLE 1. Neptune $\mathrm{CO}_{2}$ laser system.

\begin{tabular}{lcc}
\hline Parameter & Present Value & Value After Upgrade \\
\hline Wavelength & $9.2-10.8 \mu \mathrm{m}$ & $9.2-10.8 \mu \mathrm{m}$ \\
Laser Power & $1 \mathrm{TW}$ & $1-3 \mathrm{TW}$ \\
Front End Laser Power & $0.01 \mathrm{GW}-0.4 \mathrm{~Hz}$ & $10 \mathrm{GW}-1-5 \mathrm{~Hz}$ \\
Laser Pulse Length (FWHM) & $100-400 \mathrm{ps}$ & $3-400 \mathrm{ps}$ \\
\hline
\end{tabular}

TABLE 2. Neptune Photoinjector.

\begin{tabular}{lcc}
\hline Parameter & Present Value & Value After Upgrade \\
\hline Laser Energy (at 266 nm) & $0.1 \mathrm{~mJ}$ & $1 \mathrm{~mJ}$ \\
Laser Pulse Length (FWHM) & $7-10 \mathrm{ps}$ & $7-10 \mathrm{ps}$ \\
Beam Energy & $14 \mathrm{MeV}$ & $14 \mathrm{MeV}$ \\
Energy Spread & $0.2 \%$ & $0.2 \%$ \\
Electron Bunch Length (RMS) & $4 \mathrm{ps}$ & $4 \mathrm{ps}$ \\
with Chicane compression & $1 \mathrm{ps}$ & $0.2-0.5 \mathrm{ps}$ \\
Normalized Emittance & $5 \mathrm{~mm} \mathrm{mrad}$ & $2 \mathrm{~mm} \mathrm{mrad}$ \\
Charge & $-0.3 \mathrm{nC}$ typical & $0.2-2 \mathrm{nC}$ \\
Charge Stability: shot-to shot & $20 \%$ & $2-5 \%$ \\
:long term over 8 hours & $100 \%$ & $2-5 \%$ \\
\hline
\end{tabular}

We propose to organize a step-by-step replacement of the $1 \mu \mathrm{m}$ master oscillator and an amplifier (Driver) for the Photoinjector as well as the high-pressure $\mathrm{CO}_{2}$ regenerative amplifier in $\mathrm{CO}_{2}$ laser system. The former will improve both shot-to-shot and long term stability of the electron beam by more than an order of magnitude and produce a high-current ( $\sim 200 \mathrm{~A}$ ) beam using our robust, low-maintenance $\mathrm{Cu}$ cathode. The latter will increase the output from the $\mathrm{CO}_{2}$ laser system front end by a factor of 3-5 at a higher pulse repetition frequency facilitating the alignment and timing procedures. The proposed $1 \mu \mathrm{m}$ oscillator upgrade automatically makes available 14 
ps pulses from the Slicer laser. This will allow switching and amplification of a $20 \mathrm{ps}$ $\mathrm{CO}_{2}$ laser pulse and potentially 3 ps pulses in a final amplifier to a $\sim \mathrm{TW}$ and 1-3 TW level, respectively using the coherent amplification technique [5].

\section{NEPTUNE EXPERIMENTAL PROGRAM}

The primary goal for the Neptune Laboratory is to inject high quality electron bunches into a laser-driven, high-gradient (0.1-3 GeV/m) accelerating structure and explore ideas for extracting a high-quality $\Delta \mathrm{E} / \mathrm{E}<0.1$, low-emittance $<10 \pi \mathrm{mm}$-mrad, high energy (50-100 MeV) beam from such a structure. The beatwave scheme is chosen for the second generation plasma accelerator experiments [7] and we refer a laser-plasma accelerator producing a monoenergetic beam a "plasma LINAC". By now we have accelerated the injected electrons in a PBWA operating at about $1 \mathrm{THz}$ and in an IFEL accelerator driven by a high-power $\mathrm{CO}_{2}(28.3 \mathrm{THz})$ laser beam. Main results are described below.

\section{Main Experimental Results}

In the PBWA experiment a two-wavelength, 10.3 and $10.6 \mu \mathrm{m}$ laser pulse (with frequencies $\omega_{1}, \omega_{2}$ ) resonantly drives a relativistic plasma wave (RPW) of frequency $\omega_{\mathrm{p}} \approx \Delta \omega=\omega_{1}-\omega_{2}$ at the resonant plasma density $9.4 \times 10^{15} \mathrm{~cm}^{-3}$. Here for the first time a single electron bunch, with a duration shorter than the RPW's envelope, is deterministically synchronized with the RPW. For efficient injection and extraction of a high quality beam the ideal accelerating field structure would be a one dimensional plasma wave with transversely uniform accelerating fields and minimum focusing/defocusing forces. Such a 1-D structure was achieved by focusing a laser beam to a spot size of a diameter $400 \mu \mathrm{m}$; larger than the plasma wavelength $\lambda_{\mathrm{p}}=340$ $\mu \mathrm{m}$. However, if the laser itself creates the plasma, the interaction length between the electrons and the RPW is limited by ionization-induced defocusing of the laser beam [3]. We found that the interaction length and therefore energy gained by particles is enhanced significantly by using an asymmetric (fast front and slow fall) laser pulse. The duration of the pulse should be longer than the characteristic time for ions to move transversely a distance equal to $\mathrm{w}_{0}$ given by

$$
\Delta t_{i}=\frac{2 \pi w_{0} c}{e} \sqrt{\frac{m_{e} m_{i} \varepsilon_{0} c}{2 I \lambda^{2}}}
$$

where $m_{i}$ the ion mass and $I$ is the laser intensity. In this case the laser pulse is selfguided in a ponderomotively formed plasma channel. Guiding compensates for the plasma defocusing and efficiently drives the beatwave over the several-centemeterlong channel. As shown in Fig. 3(a) channel-assisted PBWA has increased the energy gain by a factor of 3.8 (from 10 to $38 \mathrm{MeV}$ ) in comparison with the case when laser pulses of the same power are shorter than $\Delta \mathrm{t}_{\mathrm{i}}$ and the plasma channel is not produced.

A collinear Thomson scattering diagnostic system was developed to study RPWs in a wide range of plasma densities, from $2 \times 10^{15}$ to $10^{17} \mathrm{~cm}^{-3}$ [8]. It is shown experimentally that RPWs in PBWA at highly nonresonant conditions when 
$\omega_{\mathrm{p}}>>\Delta \omega=\omega_{1}-\omega_{2}$ are always excited at the driving frequency $(\Delta \omega)$ and significant electrical fields of $\sim 200 \mathrm{MV} / \mathrm{m}$ could be achieved [9]. It is interesting that, according to $2 \mathrm{D}$ simulations, the accelerating fields associated with the nonresonant plasmons are always in phase with the beat-pattern of the laser pulse. This phenomenon may find an application for phase-locked acceleration of a prebuched electron beam [10].
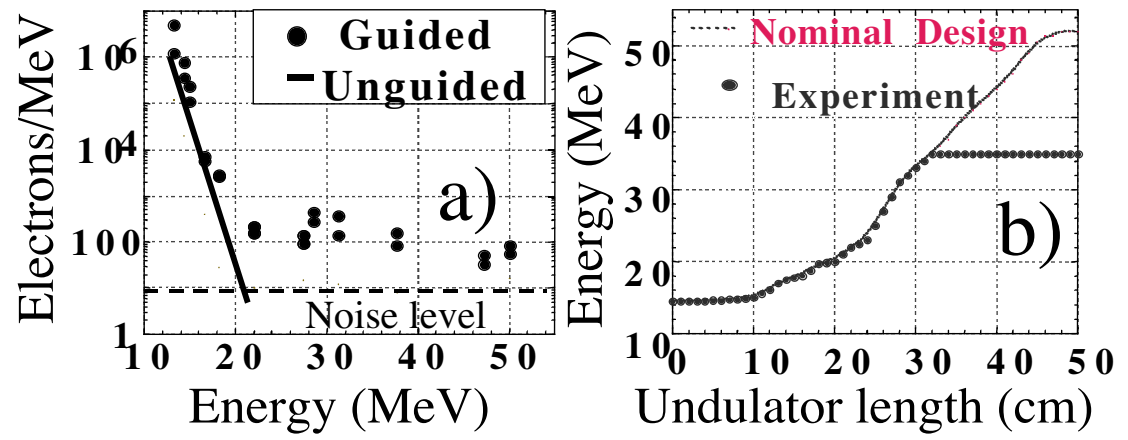

FIGURE 2. (a) Spectrum of electrons accelerated in PBWA. (b) Calculated dependence of the energy gain versus the undulator length in the $10 \mu \mathrm{m}$ IFEL experiment.

In the Neptune IFEL experiment, a $14.5 \mathrm{MeV}$ electron beam was injected in an undulator strongly tapered in period and field amplitude [4]. Precise tapering is critical to maintain the same relative phase between the electron wiggling motion and the EM wave of laser while electrons gain kinetic energy. As shown in Figure 3(b), the electrons were accelerated up to $35 \mathrm{MeV}$. Three dimensional simulations, in good agreement with the measured electron energy spectrum, indicate that most of the acceleration occurs in the first $30 \mathrm{~cm}$ of the undulator, corresponding to an energy gradient larger than $70 \mathrm{MeV} / \mathrm{m}$. This is the first experiment on a laser-driven vacuum accelerator when the acceleration gradient is larger than gradients for common RF based structures. To achieve this gradient the intensities in the laser focus reached $2 \times 10^{14} \mathrm{~W} / \mathrm{cm}^{2}$ with the Rayleigh range at about $2 \mathrm{~cm}$ and the IFEL interaction is diffraction dominated. As observed in the experiment for such coupling of the laser beam into the structure the damage threshold of the last optical element becomes a fundamental limitation for $Z_{R}$ scaling especially when the laser pulses are $200 \mathrm{ps}$ long.

A well known way to increase the interaction length between the electrons and the focused laser beam is guiding. At the laboratory the first experiments on guiding of the high-power $\mathrm{CO}_{2}$ laser pulse in a hollow waveguide both for gas-filled and evacuated conditions at intensities around $10^{14} \mathrm{~W} / \mathrm{cm}^{2}$ have been conducted [11]. The experimentally demonstrated guiding of intensities sufficient for field ionization of $\mathrm{H}_{2}$ over $2 \mathrm{~cm}\left(10 \mathrm{Z}_{\mathrm{R}}\right)$ points to the possibility to extend the PBWA length significantly. Lower, $\sim 10^{12} \mathrm{~W} / \mathrm{cm}^{2}$ intensities were guided in a $25-\mathrm{cm}$ long Ag/AgI coated hollow glass waveguide (Polymicro Inc.) in the air. We observed that for a hollow waveguide the measured energy transmission of $\sim 15 \%$ is limited by the plasma formed on the entrance surface of the waveguide which closes the coupling aperture on the time scale comparable with the $\sim 200$ ps pulse length. Clearly utilization of 20 ps pulses $\mathrm{CO}_{2}$ laser pulses should result in much higher transmission. 


\section{Future Experiments}

For the "plasma LINAC" experiment the injected electron beam should be tightly focused $\left(\sigma_{\mathrm{rms}}<30 \mu \mathrm{m}\right)$ and longitudinally prebunched at the period of RPW with a bunch length of $\lambda_{\mathrm{p}} / 7<\sigma_{\mathrm{Z}}<\lambda_{\mathrm{p}} / 4$ [10]. A low-charge, $16 \mathrm{pC}$ bunch can be compressed with a chicane to $\sigma_{Z} \sim 75 \mu \mathrm{m}$ [6] which is less than $\lambda_{\mathrm{p}} / 4$ for the $340 \mu \mathrm{m}$ wavelength of the plasma structure. This would allow for the placement of all $\sim 10^{8}$ electrons within the accelerating and focusing bucket of the RPW, albeit with a shot-to-shot phase jitter on the $\mathrm{THz}$ scale of $100 \%$. Although we consider this step as a possible proof-ofprinciple experiment, improvement in the beam charge stability (at least to $<5 \%$ ) becomes a critical issue. Ultimately a controlled injection of the electron beam in the PBWA requires $\mathrm{THz}$ microbunching that is phase-locked to the RPW phase.

There are several ways for microbunching(in vacuum) phase-locked to the PBWA : 1) $\mathrm{THz}$ IFEL microbunching is based on resonant energy exchange from the highpower EM wave to the electron beam propagating collinearly in a short undulator. Recently we proposed to produce high-power $340 \mu \mathrm{m}$ radiation by difference frequency generation (DFG) in a nonlinear crystal, mixing the same $\mathrm{CO}_{2}$ laser lines that are used for RPW excitation [10]. Thus phase-locking could be achieved. According to detailed 3D modeling a THz beam with an intensity of $6 \mathrm{MW} / \mathrm{cm}^{2}$ guided over $0.5 \mathrm{~m}$ inside the undulator is sufficient for microbunching. Preliminary results on DFG using 200 ps $\mathrm{CO}_{2}$ laser pulses have shown that generation of $\mathrm{THz}$ radiation at $\mathrm{MW}$ power level will require a large-aperture multi $\mathrm{GW} \mathrm{CO}_{2}$ laser beam of high quality to pump a GaAs crystal with diameter of 5" without optical damage.

2) THz FEL microbunching utilizes the resonant energy exchange from the electron beam to a seed EM wave inside an undulator. For a high-gain FEL less than $100 \mathrm{~W}$ of $\mathrm{THz}$ power was sufficient for seeding [12]. As opposed to using a separate THz FEL oscillator suggested for an optical seed production in [12], we consider injecting in a waveguide $340 \mu \mathrm{m}$ FEL (peak current $>100 \mathrm{~A}$ ) a kW THz pulse produced by DFG.

3) Laser beatwave injection of a FEL, as suggested by G. Shvets [13], is based on nonlinear beatwave interaction of the TW lasers with the beam inside an undulator. Via $\mathrm{THz}$ modulation of the beam, production and amplification of $\mathrm{THz}$ noise radiation occurs according to the SASE FEL mechanism. This eventually results in a seedless bunching of electrons on the laser beatwave scale. However, the interaction length for optimal bunching of a $14 \mathrm{MeV}$ beam should be of the order of 6 meters [13].

4) Laser beatwave ponderomotive microbunching is using a very high ponderomotive force attributed to a focused $\mathrm{TW} \mathrm{CO}_{2}$ laser beam interacting with an electron beam in vacuum [14]. For this experiment $2 \mathrm{TW} \mathrm{CO}_{2}$ laser pulses are desirable.

In general we want to study microbunching with and without production of a $\mathrm{THz}$ seed radiation. However, at the moment an FEL-buncher looks advantageous, since only part of the laser beam is needed for generating the $\mathrm{kW}$ seed pulse. We also plan to model injection of both the $\mathrm{THz}$ seed pulse and the laser beatwave into the FEL buncher to study mechanisms that may increase the efficiency for beam modulation. Note that modulation of the electron beam on the $\mathrm{THz}$ scale is of interest for the beam physics since direct measurements of longitudinal picosecond dynamics are possible.

In the $10 \mu \mathrm{m}$ IFEL program at the Neptune Laboratory we plan to focus on a further increase in the energy gain obtained in a single stage accelerator. One possible 
scenario is a $80 \mathrm{~cm}$ long helical IFEL where a $\mathrm{CO}_{2}$ laser beam with a $5 \times 10^{13} \mathrm{~W} / \mathrm{cm}^{2}$ intensity is guided in a hollow waveguide and electrons are accelerated from 14 to $100 \mathrm{MeV}(\sim 120 \mathrm{MV} / \mathrm{m})$ [15]. It is obvious that efficient guiding over almost a meter long distance is a prerequisite for such an experiment. Production of shorter $10 \mu \mathrm{m}$ pulses after the proposed upgrade will facilitate guiding in $0.1-1 \mathrm{~m}$ long waveguides.

Guiding of the intense laser beatwave pulse $\left(10^{14}-10^{16} \mathrm{~W} / \mathrm{cm}^{2}\right)$ in a $10 \mathrm{~cm}$ long hollow waveguide filled with a gas will make possible a PBWA in which the interaction length approaches the dephasing length, i.e. the distance over which the electrons overtake the plasma wave and stop gaining energy. For example, for a resonant density of $10^{16} \mathrm{~cm}^{-3}$ the energy gain lies in $100 \mathrm{MeV}$ to $300 \mathrm{MeV}$ range for the $10 \%$ and $30 \%$ plasma wave amplitude, respectively. More breathtaking $\sim 1 \mathrm{GeV}$ energy gain for injected electrons is achievable by switching to 2 ps pulses at 1.05 and $1.06 \mu \mathrm{m}$ [16] and the plasma density of $10^{17} \mathrm{~cm}^{-3}$. It is important that the radial dependence of the RPW in a gas-filled waveguide can be mainly homogeneous: the plasma is created by gas ionization and not related to guiding itself. Natural first step towards a guided PBWA will be a measurement of RPW inside the waveguide using the Thomson scattering diagnostic system [8].

In the near future we continue to work on a longitudinal electron beam shaping using the "S-bahn" [17]. On a part of the Neptune program dedicated to development of laser-based light sources we plan to study the production of polarized X-rays via Compton scattering [18] and to optimize the process of $\mathrm{THz}$ generation using DFG.

\section{ACKNOWLEDGMENTS}

This work was supported by U.S. DoE, grant DE-FG03-92ER40727.

\section{REFERENCES}

1. Joshi, C and Katsouleas, T., Physics Today, 56, 47 (2003).

2. Kimura, W. D., et al., Phys. Rev. STAB 4, 101301 (2001).

3. Tochitsky, S. Ya., et al., Phys. Plasmas. 11, 2875 (2004).

4. Musumeci, P., et al., in this Proceedings.

5. Tochitsky, S. Ya., et al., Opt. Lett. 24, 1717 (1999). Tochitsky, S. Ya., et al., ibid. 26, 813 (2001).

6. Anderson, S. G., et al., Advanced Accelerator Concepts, edited by P.L. Colestock, AIP Conference Proceedings 569, Melville, New York, 2001, pp. 487-489.

7. Clayton, C.E., et al., NIMP A 410, 378 (1998).

8. Filip, C. V., et al., Rev. Sci. Instrum. 74, 3576 (2003).

9. Filip, C. V., et al., Phys. Rev. E 69, 026404 (2004).

10. Tochitsky, S. Ya., et al., Advanced Accelerator Concepts, edited by C.E. Clayton and P. Muggli, AIP Conference Proceedings 647, Melville, New York, 2002, pp. 786-795.

11. Sung, C., et al., in this Proceedings.

12. Lampel, M., et al in Proceedings of the 1995 Particle Accelerator Conference, Dallas, TX, p. 764.

13. Shvets, G., "Beatwave injection of a Free-Electron Laser" unpublished.

14. Gordon, D.F., et al., Phys. Rev. E 57, 1035 (1998).

15. Rosenzweig, J. B., et al., in this Proceedings.

16. Hankla, A. K., et al., Opt. Lett. 22, 1713 (1997).

17. England, R. J., et al., in this Proceedings.

18. Doyuran, A., et al., in this Proceedings. 